\title{
Aesthetic Correction of a Protrusive Forehead through Repositioning of the Anterior Wall of the Frontal Sinus
}

\author{
Daniel Seungyoul Han', \\ Jin Hyung Park ${ }^{2}$ \\ ${ }^{1}$ Department of Craniofacial Center, Chang \\ Gung Memorial Hospital, Taoyuan, Taiwan; \\ ${ }^{2}$ Department of Plastic and Reconstructive \\ Surgery, Kosin University Gaspel Hospital, \\ Busan, Korea
}

No potential conflict of interest relevant to this article was reported.
Facial skeletal remodeling was revolutionized more than 30 years ago, by the work of Tessier and other craniofacial surgeons. However, the need to correct the skeleton in the upper third of the face is not frequently diagnosed or treated in aesthetic facial surgery. Here, we report on the aesthetic correction of a protrusive forehead. A patient visited our hospital for aesthetic contouring with a prominent forehead. The anterior wall of the frontal sinus was removed with a craniotome via the bicoronal approach. After the excised bone was repositioned, it was fixed with a titanium mesh plate and screws. An electric burr was used to contour the supraorbital rim and frontal bone. Once the desired shape was achieved, the periosteum was replaced, and the wound was closed in layers. When performed properly, frontal sinus contouring could significantly improve the appearance in patients with a prominent forehead. Plastic surgeons must carefully evaluate patients with a prominent forehead for skeletal remodeling that involves the accurate and safe repositioning of the anterior wall of the frontal sinus.

Keywords: Prominent forehead / Facial feminization surgery

\section{INTRODUCTION}

Skeletal reconstruction and contouring of the forehead or upper face cannot be surgically managed in the same manner that lower facial skeletons are manipulated. Thus, patient satisfaction with outcomes is an issue. However, these surgical modalities are rarely performed. Yet, protruding forehead is quite common and typically givean excessively masculine or obstinate appearance. Patients with such forehead feature express certain difficulty with interpersonal relationships due to misunderstanding or discomfort [1].

At our medical institution, idiopathic frontal protrusion is corrected by removing the anterior wall of the frontal sinus via a bi-

Correspondence: Daniel Seungyoul Han

Department of Craniofacial Center, Chang Gung Memorial Hospital,B1, No.123, Dinghu Road, Gueishan, Taoyuan (333), Taiwan

E-mail: doc6029@naver.com

Received September 23, 2014 / Revised November 15, 2014 /

Accepted December 5, 2014 lateral frontal approach. This anterior wall is spread, re-arranged with a titanium mesh plate and screws, after which the new anterior wall contstruct is fixed to the adjacent frontal bone. Using this surgical technique, we have successfully treated patients with frontal protrusion. Here, we report our experience along with a literature review.

\section{CASE REPORT}

A 28-year-old man visited us with the chief complaint of a markedly protruding forehead. The patient wished for a surgical correction because he often experienced awkward social interactions (Fig. 1A). Radiologically, the forehead was seen to have protrusion and extension with hyperpneumatization of the frontal sinus (Fig. 2A). Prior to the operation, the patient had a frontal slope of $19^{\circ}$, confirming the need for surgical correction in this case (Fig. 1A).

A bilateral coronal incision was made following the general de- 

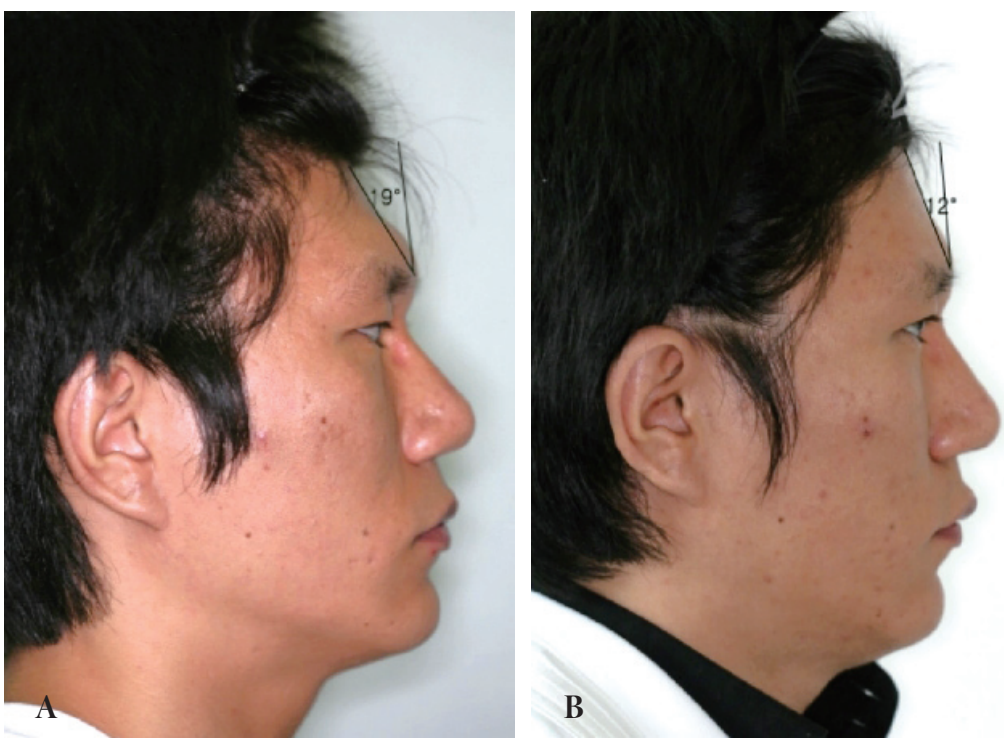

Fig. 1. (A) Preoperative forehead inclination was an angle of $19^{\circ}$ from trichion to glabella. (B) Postoperative forehead inclination has formed an angle close to the average male.
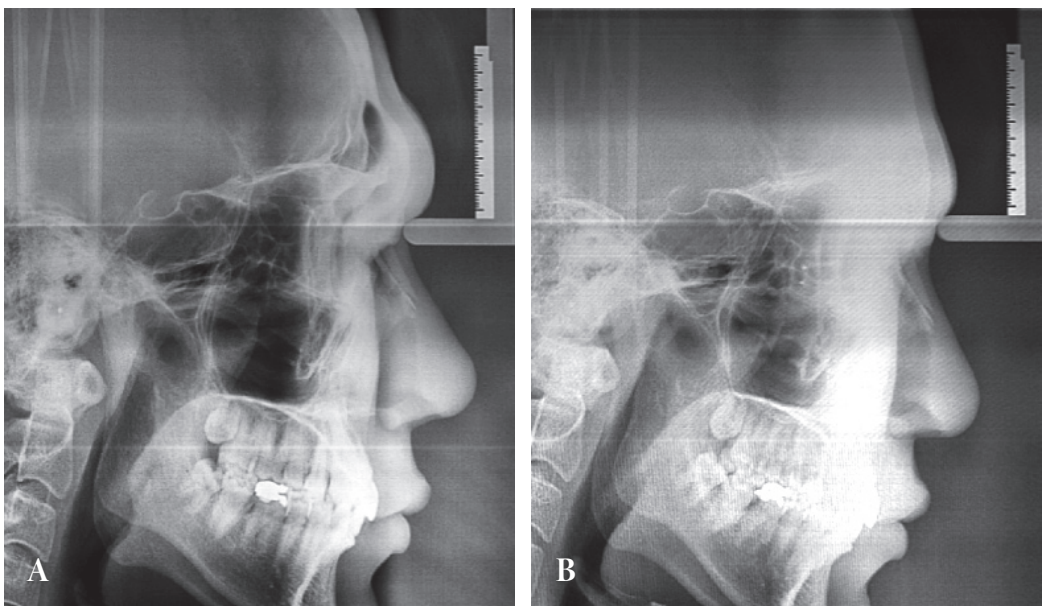

Fig. 2. (A) Preoperative lateral view of cephalogram. Prominent anterior wall of frontal sinus was noted. (B) Postoperative lateral view of cephalogram. The prominence of anterior wall of frontal sinus was reduced.

sign. The dissection was performed up to and including the supraorbital crest and the periosteum of the external supraorbital margin on both sides. The periosteum was incised $2 \mathrm{~cm}$ superior to the supraorbital margin. As a result, the dissection included the medial side of the supraorbital margin. After an interoperative skull anteroposterior X-ray was used to determine the extent of frontal sinus, a landmark was denoted with a pencil. The frontal sinus was entered at the landmark using an electrical burr. The anterior wall of the frontal sinus was removed using a craniotome; the posterior wall and nasofrontal canal were remained un- touched. The excised anterior wall was spread completely with a bone bender, and re-arranged using a titanium mesh plate. Thus, the frontal contour was corrected without any functional impairment of the frontal sinus.

Following this, the margins of anterior wall construct and protruding supraorbital rim were trimmed with an electrical burr. The construct was then secured to the adjacent cortical bone with titanium screws. By maximally preserving a pericranial flap, we were able to prevent bone resorption. The scalp flap was closed in layers, and the overall contour of the forehead was confirmed (Fig. 3). 


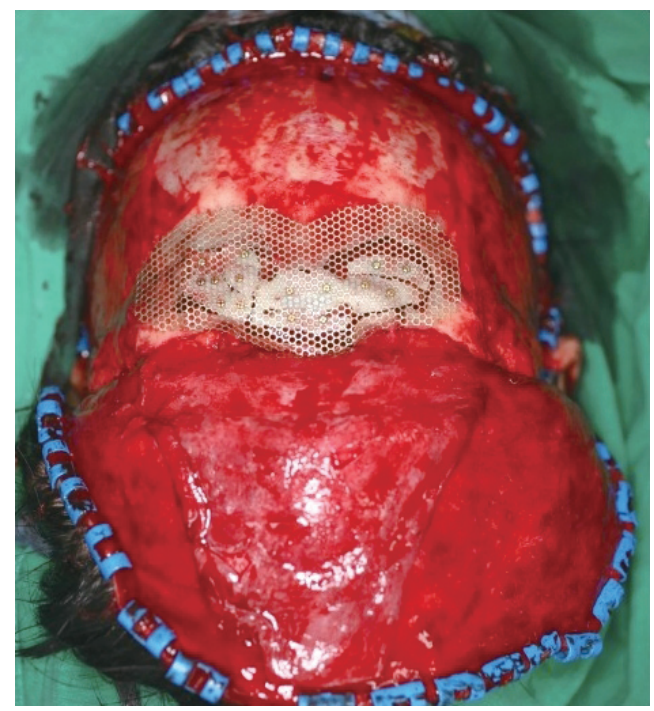

Fig. 3. The anterior wall of frontal sinus was remodeled by repositioning and fixation with mesh plate and screws.

The postoperative slope angle of the forehead was $12^{\circ}$, which was $7^{\circ}$ less than the preoperative measurement (Fig. 1B). The patient was able to resume activities of daily life, including hair washing, on the first day after operation. At postoperative week 3 , cephalometry revealed a flatter forehead (Fig. 2B). During the 36month follow-up period, there were no signs of infection such as chronic frontal sinusitis.

\section{DISCUSSION}

In general, the facial region can be divided into three parts. The vertical distance between the nasale and trichion defines the upper part of face [2]. This area is an important cosmetic unit as it includes the eyes and forehead, which greatly affect the first impression of a person. In other words, the forehead and supraorbital margin play a crucial role in defining masculine and feminine facial features. From the perspective of cephalometry, the mean slope angle is approximately $10.5^{\circ}$ in men and $7^{\circ}$ in women. This suggests that a greater degree of frontal slope angle contributes to a more masculine facial feature [2].

In patients with a protruding forehead without functional problems, the following cases are suitable for surgical treatment [3]: 1) a marked protrusion of the supraorbital area and forehead in anatomical relationship to the eyeball; 2) an inferior displace- ment of the overall area of the supraorbital margin; and 3) a lateral protrusion of the orbital margin.

To completely reconstruct the protruding portion of forehead in whole, we removed the anterior wall of protruding frontal sinus and reconfigure the plate with titanium mesh plate. Although anterior sinus wall is not a weight bearing structure, we used a titanium mesh plate and screws for fixation because of cost-efficiency and thin profile when compared to absorbable plate systems [4]. For reconstruction of post-traumatic or tumor-resection defects of the anterior wall of frontal sinus, removal and reshaping of the anterior wall has been suggested. Particularly, a titanium mesh plate can be used to successfully perform the reconstructive surgery without minimal complications where the defect area is large or the bone fragments cannot be used due to the presence of comminuted fracture [3,5]. This eventually provided the basis on which cosmetic contouring surgery could be performed, especially in patients with a protruding forehead. As a matter of fact, Whitaker et al. [6] performed a dissection of the anterior frontal sinus wall based on the safety of reconstruction operation and subsequently reposition the wall in a more posterior location. Viterbo et al. [7] reported fixing the anterior wall using wires. There are technical differences among the cosmetic surgery methods used for protruding forehead. Nevertheless, the above reports have confirmed that it is safe to remove the anterior frontal sinus wall in performing contouring surgery with minimal complications. Moreover, patients who undergo frontal contouring can quickly resume daily activities after the surgery.

\section{ACKNOWLEDGMENTS}

This study would not have been possible without supporting of my family. The author wishes to express my love and gratitude to my beloved families; for their understanding \& endless love, through the duration of my studies.

\section{REFERENCES}

1. Urken ML, Som PM, Lawson W, Edelstein D, Weber AL, Biller HF. Abnormally large frontal sinus. II. Nomenclature, pathology, and 
symptoms. Laryngoscope 1987;97:606-11.

2. Bartlett SP, Wornom I 3rd, Whitaker LA. Evaluation of facial skeletal aesthetics and surgical planning. Clin Plast Surg 1991;18:1-9.

3. Sykes JM, Moore EJ. Esthetic contouring of the forehead and supraorbital rims. Facial Plast Surg Clin North Am 2002;10:303-10.

4. Gosain AK, Song L, Corrao MA, Pintar FA. Biomechanical evaluation of titanium, biodegradable plate and screw, and cyanoacrylate glue fixation systems in craniofacial surgery. Plast Reconstr Surg 1998; 101:582-91.
5. Lakhani RS, Shibuya TY, Mathog RH, Marks SC, Burgio DL, Yoo GH. Titanium mesh repair of the severely comminuted frontal sinus fracture. Arch Otolaryngol Head Neck Surg 2001;127:665-9.

6. Whitaker LA, Morales L Jr, Farkas LG. Aesthetic surgery of the supraorbital ridge and forehead structures. Plast Reconstr Surg 1986;78: 23-32.

7. Viterbo F, Ranzani F, Campos E. New treatment for frontal sinus hypertrophy. Plast Reconstr Surg 1990;86:776-9. 